\title{
Neural Decoding and Applications in Bioelectronic Medicine
}

\author{
Chad Bouton \\ Battelle Memorial Institute, Columbus, Ohio, United States of America
}

\begin{abstract}
Neural decoding is a field involving the use of signal processing and machine learning methods to decode brain activity for various applications including assistive technology for people living with paralysis and diagnosing brain-related diseases such as Parkinson's, Alzheimer's, schizophrenia and obsessive compulsive disorder. The use of neural decoding, however, could be extended to applications in bioelectronic medicine, a field focused on the treatment and diagnosis of organ and other diseases through neurostimulation and neurosensing in the central and peripheral nervous system. Specifically, there is increasing evidence that neurostimulation can upregulate or downregulate the immune system, and, just as the nervous system innervates our organs and helps regulate their function, it also can modulate the immune system response and even affect acute inflammation response. Previous research in bioelectronic medicine has been focused primarily on open-loop neurostimulation without sensing methods or algorithms to automatically control the spatial and temporal characteristics of the stimulation. Research involving neural decoding methods has been conducted for other neurostimulation applications including the treatment of epilepsy and Parkinson's disease. Introducing sensing and neural decoding methods into the bioelectronic medicine field could improve the diagnosis and treatment of a wide variety of diseases by closing the loop, which would allow automatic and adaptive neurostimulation that could increase its overall efficacy. There will be important related research questions and challenges to address as we attempt to place a control system on top of an already existing control system—a vast, complex and dynamic one_the human nervous system.
\end{abstract}

Online address: www.bioelecmed.org

doi: 10.15424/bioelectronmed.2014.00012

\section{INTRODUCTION}

Neural decoding has primarily been a field that focuses on deciphering neural activity to extract useful information about sensory, motor or even disease states within the brain, but could potentially be expanded to the entire nervous system in the future. One area within this field that has gained significant attention is neural decoding of brain activity in patients living with paralysis, and/or patients who cannot speak, to allow control over devices such as computers, robotic arms or an electric wheelchair through the patient's thoughts alone. Much focus has been placed on neural activity within the brain; however, neural decoding methods also may be applied to other parts of the nervous system to improve the diagnosis and treatment of neurological injury and, potentially, a wide range of diseases.

Bioelectronic medicine is a seemingly separate field that involves electrical stimulation in the nervous system (neurostimulation) with the primary goal of treating organ and immune system diseases. Neurostimulation in the broader view has many benefits including pain management, lowering blood pressure, promoting nerve and bone growth, wound healing and treating diseases such as Parkinson's, Alzheimer's, schizophrenia, obsessive compulsive disorder, obesity and immune system-related disorders such as rheumatoid arthritis.

Address correspondence to Chad Bouton, Battelle Memorial Institute, 505 King Ave., Columbus, OH 43201. Phone: 614-424-7910; Fax: 614-458-7910; E-mail: bouton@battelle.org. Submitted December 10, 2014; Accepted for publication January 22, 2015; Published Online (wWw.bioelecmed.org) January 22, 2015.

\section{The Feinstein Institute for Medical Research Empowering Imagination. Pioneering Discovery.}

However, in many cases, neurostimulation is applied in an open-loop fashion, meaning that it is manually adjusted and feedback is not provided from sensed parameters such as neural signals or other biomarkers. Lacking sensed parameters, automatic adjustment (that is, closed-loop control) is not possible for adapting the stimulation as the patient's symptoms improve or worsen or to assist in the initial adjustment of stimulation parameters.

Combining ideas from the two fields of neural decoding and bioelectronic medicine could significantly increase the efficacy of diagnosis and treatment of a wide range of neurological conditions and disease. Neural decoding methods can detect and identify patterns often difficult or impossible to recognize otherwise, and can be a powerful tool for researchers and physicians in diagnosis or followup. Furthermore, neural decoding algorithms can be embedded in the medical devices being developed for the bioelectronic medicine field and improve their effectiveness. As we move forward, there are many remaining research questions and technical challenges to maxi- 


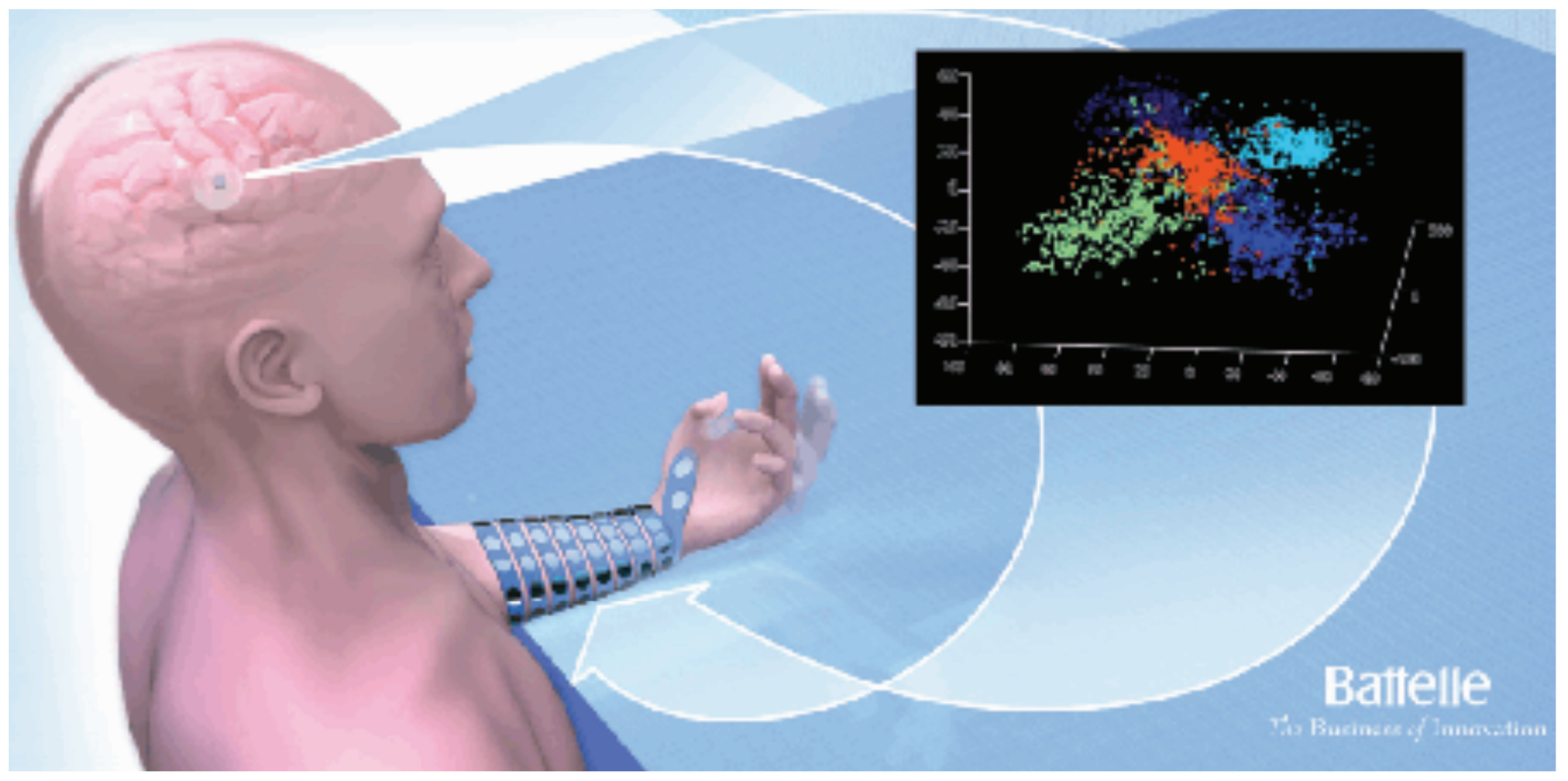

Figure 1. Neural bridging concept: linking neural decoding to neurostimulation-driven movement in a paralyzed user (courtesy of Battelle).

mize the benefit of merging methods from the fields of neural decoding and bioelectronic medicine, however, early progress suggests we are heading in the right direction.

\section{SIGNIFICANT ADVANCEMENTS AND REMAINING CHALLENGES}

There have been significant historical and recent advancements in the neural decoding and bioelectronic medicine fields. Early research in neural decoding involved recording and analyzing motorand sensory-related activity in the brain to understand how information is encoded $(1,2,3,4,5)$. Details about neuronal firing patterns in response to various stimuli or changes in motor actions were studied. Ideas such as directional 'tuning' were introduced where neuronal firing increased in the motor cortex, for example, as arm movement occurred in certain spatial directions (6). Further development in neural decoding and implant technology led to studies in humans involving patients living with impairments from spinal cord injury, stroke or amyotrophic lateral sclerosis (ALS) $(7,8,9)$. In these studies, neural decoding methods were developed to decipher and classify neural patterns in the motor cortex, which allowed patients to control devices such as a computer, robotic arm or a powered wheelchair $(10,11,12,13)$. As the patient imagined/attempted various arm and hand movements, neural decoding algorithms were developed and trained to automatically recognize the neural activity patterns associated with each movement or direction of movement desired. Once an effective decoding algorithm was in place, patients could use their thoughts to control a cursor on a computer or to control various devices with their thoughts alone. This further expanded the area of brain-computer interfaces. Some limitations are associated with the neural implant portion of brain-computer interfaces, however, which includes potential degradation of neural signals, particularly single unit activity, over extended periods of time (months/years) during chronic implantation $(14,15)$.

Nonhuman primate work that involves linking intracortical brain activity to actual movement has been performed previously as well $(16,17)$. In these studies, neural decoding has been combined with neuromuscular stimulation to allow volitional control of temporarily paralyzed muscles. Most recently, the first demonstration of linking neural decoding of intracortical signals to neuromuscular stimulation in a human has been shown during a clinical study where a 23-yearold male quadriplegic participant moved his paralyzed hand under volitional control (18). As shown in Figure 1, this concept, called "neural bridging," was demonstrated which involves decoding signals from a cortical implant and linking them to high-resolution neuromuscular stimulation. This allows a paralyzed user to regain continuous, volitional wrist and dexterous finger movements through their own thoughts.

Cortical implants provide significantly higher temporal resolution than functional magnetic resonance imaging (fMRI) and high spatial resolution as well. For example, commercially available electrode arrays can have electrodes spaced as closely as 200 to 400 microns center-to-center, and the sampling rates of commercially available systems can be up to $30 \mathrm{kHz}$. High spatial and temporal resolution allows detailed study of the neural signals being recorded by the electrode array. This also facilitates the development of neural decoding algo- 
rithms and methods since various features in the neural signals, such as single unit firing rate, multi-unit activity levels and synchrony, can be obtained and used for algorithm training. One significant limitation that currently exists, however, is the potential for signal degradation in a neural implant when sensing/recording signals over an extended period of months/years $(14,15)$. Issues involve implant mechanical/material issues such as insulation breakdown and biological issues such as degeneration of neurons near the electrode surface and encapsulation. Using signal processing methods to extract amplitude information related to multiple neuronal signals, known as multiunit activity, provide a useful set of features for decoding that can be more robust over time $(19,20,21)$.

Reliable neural interfaces for sensing/recording over time will be required to develop closed-loop, commercially available bioelectronic systems for patients. Furthermore, adapting the current electrode array-type neural implants (mostly targeted for cortical applications) for peripheral nerves will be important. Also, higher resolution neural implants (electrode density) with more electrodes may be required to gain a detailed and complete picture for closed-loop neurostimulation systems (on the sensing side) to treat various conditions of interest. This will depend on the complexity of the peripheral nervous system circuit of interest, and the specificity required for stimulation will drive the stimulating electrode requirements.

A key step in neural decoding is to identify features in the signals, such as firing rate in action potentials, multiunit activity, or correlation/coherence between pairs of signals being monitored, for example. Another key step is to use pattern recognition, or machine learning methods, to translate the signals into a form that is useful and correlates with motor- or sensory-related events. For example, if a paralyzed patient is asked to imagine or attempt a movement, using machine learning methods will allow the patterns to be 'learned' by the decoding algorithm and recognized automatically in the future, based solely on the neural activity being monitored by the sensor/electrode array being used. This idea could be utilized in the peripheral nervous system as well to recognize neural signatures that correlate with various disorders or conditions. Furthermore, patterns associated with the related symptoms as they improve or worsen could potentially be learned by neural decoding algorithms and tracked over time. This could allow a closed-loop system to be designed to provide automatic control and adaptation of the stimulation.

Neural sensing/recording and subsequent decoding also could be used as a diagnostic tool and/or to help identify patients who would be good candidates for neurostimulation in their particular condition. Neural decoding methods have been used to identify brain activity through MEG (magnetoencephalography) associated with various disorders including multiple sclerosis, Alzheimer's disease, schizophrenia, Sjögren's syndrome, chronic alcoholism and facial pain (22). This work involved creating features from the MEG data that represented important attributes of the neural networks within the brain such as dynamic synchrony. These features are then input into machine learning algorithms, which can aid in classifying patients with different disorders and those who are healthy as well. With sufficient sensing points in the peripheral nervous system, neural decoding methods may also be able to help recognize and classify a patient's condition. In the future, noninvasive sensing and neural decoding methods combined may be able to help predict how effective stimulation will be. In addition, neural decoding methods may be utilized in guiding where the stimulation should be applied before surgical procedures are employed.

In the fields of neurostimulation and bioelectronic medicine, there are potential benefits in many areas, including chronic pain $(23,24)$, respiration/bladder/bowel/sexual function in those liv- ing with paralysis (25), epilepsy (26), high blood pressure (27), depression and obsessive compulsive disorder (28) and inflammatory diseases such as Parkinson's (29), Alzheimer's (30), Crohn's and rheumatoid arthritis (31). Specifically, there is increasing evidence that neurostimulation can upregulate or downregulate the immune system in many beneficial ways, and just as the nervous system innervates our organs and helps regulate their function, it also can also modulate the immune system response and even affect acute inflammation response (32). In one landmark study, it was shown that efferent vagus nerve signaling could modulate inflammation, and the response to endotoxin and neurostimulation of the peripheral vagus nerve during lethal endotoxemia in rats inhibited TNF (tumor necrosis factor) synthesis in the liver and prevented the development of shock (33). Furthermore, it was determined that macrophages in the spleen were making the TNF and, since the vagus nerve innervates the spleen, the stimulation facilitated the reduction of the TNF production (34).

There is one element that many of the neurostimulation studies that have been conducted previously have in common: the stimulation was applied in an openloop fashion with no neural sensing or decoding employed to gain a deeper insight into the neural signaling in the nervous system prior to and after the stimulation. There are some exceptions that have emerged where a form of neural decoding has been used with neurostimulation. These include early work in closed-loop deep brain stimulation (DBS) for Parkinson's or essential tremor and neurostimulation for seizure management. In the case of closed-loop DBS, neural signals are sensed in the brain and processed to automatically adjust the stimulation, which shows promise in improving efficacy as compared with open-loop stimulation (35). In the case of seizure management, neural activity that could lead to a seizure is identified by a neural decoding type algorithm which triggers stimulation to prevent or sup- 
press the seizure, such as in the NeuroPace RNS device. Various signal features have been studied including amplitude, coherence and nonlinear methods (36) that can be used for triggering the stimulation. Studies in cultures also have been performed to study the effects of stimulation on neuronal networks (37).

In the early research around closedloop neurostimulation, a low number of sensed signals/parameters (typically less than 10) are used and therefore the information content is relatively low, reducing the observable states within the neural network being monitored. This is a limitation that may create challenges in creating effective control algorithms.

Typically in neural decoding applications in the brain, larger sets of signals are collected (dozens to hundreds, currently) to recognize and classify relatively complex neural patterns and states in the cortex. Neural decoding could be a powerful approach for gaining deeper insight into the behavior of the peripheral nervous system and also for allowing effective closed-loop neurostimulation.

One challenge that currently exists for implementing neural decoding in the central or peripheral nervous system is obtaining a sufficient number of signals. Sensing multiple signals from neural pathways such as nerve fibers is a significant challenge and requires small implantable neural interfaces, which are still under development today. Furthermore, an interface of this nature can fail to provide quality signals over time due to biomechanical issues such as migration, encapsulation and scarring.

Finally, controlling complex systems typically requires not only multiple feedback parameters, but also multiple control inputs. In the case of neurostimulation, this means multiple stimulation points may be required within the nervous system to achieve the desired levels of efficacy across the target patient population. Just as with neural sensing, there are currently challenges and limitations in stimulating at multiple points in the nervous system. Achieving the spatial resolution and mechanical stability required are among those challenges. Also, if the stimulation targets are physically far apart, multiple incisions may be required, driving the need for smaller implants that can be installed with minimally invasive methods or high resolution transcutaneous stimulation technology.

\section{DISCUSSION}

Neural decoding has shown successes in the area of motor cortex decoding for applications in paralysis and in brain activity classification for disease diagnosis. The use of neural decoding could allow automatic recognition and classification of various organ disease states as well, or it could be used to track if symptoms are worsening or improving. This could potentially be accomplished at some point through sensing and deciphering of neural signaling that is occurring within the peripheral nervous system.

Conditions such as Parkinson's often involve an imbalance in pathways (or over/under active circuits within the brain). To better understand and diagnose these conditions, better tools are needed to monitor and characterize multiple pathways simultaneously in the central and peripheral nervous system as we further develop neurostimulation devices and treatments. Neural decoding may be very useful in the characterization process as its power lies in its ability to identify and classify different patterns that are very difficult to see in raw and/or large sets of signals.

Another remaining challenge in developing methods and devices for the field of bioelectronic medicine is to improve the maps available for the human nervous system. This mapping must be for both normal and diseased nervous systems. This will involve large data sets and require signal processing, machine learning and computational modeling methods to expand our knowledge of the vast pathways, connections and signaling within the nervous system. Furthermore, computational modeling of portions of the nervous system associated with various disorders or conditions will be very important in developing new de- vices and methods in the field of bioelectronic medicine.

Computational models should represent the normal, diseased and perturbed (stimulated) behaviors. Closed-loop control of neurostimulation in bioelectronic medicine applications will offer exciting challenges. From a control theory perspective, even when sensing at multiple points in the network of interest, the number of measured states will be significantly lower than the number of internal states of importance, which can make creating a control structure challenging. This will require utilization of computational models so that effective observers (to estimate unmeasured internal states in the network) can be developed and model-based control methods can be employed.

There are many remaining areas of debate and outstanding research questions in the fields of neural decoding and bioelectronic medicine. How information is encoded in the human nervous system is not well understood and the details of signaling and interactions in the intertwined organ and immune systems are still under study. As we attempt to control the nervous system through neurostimulation to address various diseases and conditions, we will encounter many exciting challenges. In essence we will be attempting to place a control system on top of an already existing one-a vast, complex and dynamic one-the human nervous system.

Over the next 10 to 20 years, we will see a continued expansion of bioelectronic medicine and bionic technology. We will see the continued merger of person and machine including further advancements in cochlear implants for the deaf, prosthetic arms for amputees, artificial retinas for the blind and brain-computer interfaces and neural bridging technology for the paralyzed. As we attempt to combine biology and electronics, there will continue to be many challenges. However, if successful, this may open many new doors and treatment options for a wide range of diseases which may, in turn, lead to a higher quality of 
life for the millions of people that are affected every day.

\section{REFERENCES}

1. Evarts EV (1968). Relation of pyramidal tract activity to force exerted during voluntary movement. J. Neurophysiol. 31:14-27.

2. Perkel DH, Bullock TH. (1968) Neural coding. Neurosci. Res. Program Bull. 6:221-348.

3. Georgopoulos AP, Schwartz AB, Kettner RE. (1986) Neuronal population coding of movement direction. Science. 233:1416-9.

4. Warland DK, Reinagel P, Meister M. (1997) Decoding visual information from a population of retinal ganglion cells. J. Neurophysiol. 78:2336-50.

5. Donoghue JP, Sanes JN, Hatsopoulos NG, Gaál G. (1998). Neural discharge and local field potential oscillations in primate motor cortex during voluntary movements. J. Neurophysiol. 79:159-73.

6. Georgopoulos AP, Kalaska JF, Caminiti R, Massey JT. (1982). On the relations between the direction of two-dimensional arm movements and cell discharge in primate motor cortex. J. Neurosci. 2:1527-37.

7. Kennedy PR, Bakay RAE. (1998) Restoration of neural output from a paralyzed patient by direct brain connection. Neuroreport. 9:1707-11.

8. Taylor DM, Tillery SIH, Schwartz AB. (2002) Direct cortical control of $3 \mathrm{D}$ neuroprosthetic devices. Science. 296:1829-32.

9. Hochberg LR. (2006) Neuronal ensemble control of prosthetic devices by a human with tetraplegia. Nature. 442:164-71.

10. Hochberg LR, et al. (2012). Reach and grasp by people with tetraplegia using a neurally controlled robotic arm. Nature. 485:372-5.

11. Humber C, Ito K, Bouton C. (2010) Nonsmooth formulation of the support vector machine for a neural decoding problem [Internet]. [Ithaca (NY)]: arXiv; [cited 2015 Apr 21]. Available at: http://arxiv.org/abs/1012.0958.

12. Bouton C. (2010 Oct 13). Neural decoding: what's on your mind?: breakthrough technology helps patients channel thoughts into actions [Internet]. Med. Des. [cited 2015 Apr 21]. Available from: http://medicaldesign.com/prototyping/neuraldecoding-what-s-your-mind

13. Bouton C. (2009). Decoding neural activity from an intracortical implant in humans with tetraplegia [Internet]. [cited 2015 Apr 21]. Session presented at: Biomedical Science \& Engineering Conference, March, 2009. BSEC 2009. First Annual ORNL; 2009 Mar 18-19; Oak Ridge, TN. Available from: http://ieeexplore.ieee.org/xpls/ abs_all.jsp?arnumber $=5090450$

14. McConnell GC, et al. (2009). Implanted neural electrodes cause chronic, local inflammation that is correlated with local neurodegeneration. J. Neural Eng. 6:056003.

15. Barrese JC, et al. (2013). Failure mode analysis of silicon-based intracortical microelectrode arrays in non-human primates. J. Neuro. Engin. 10:066014.
16. Moritz CT, Perlmutter SI, Fetz EE. (2008) Direct control of paralysed muscles by cortical neurons. Nature. 456:639-42.

17. Ethier C, Oby ER, Bauman BJ, Miller, LE. (2012) Restoration of grasp following paralysis through brain-controlled stimulation of muscles. Nature. 485:368-71

18. Tankersley J. (2014 Jun 24) Science fiction come true: moving a paralyzed hand with the power of thought [Internet]. Washington Post. [cited 2015 May 15]. Available from: http://www. washingtonpost.com/business/economy/sciencefiction-come-true-moving-a-paralyzed-handwith-the-power-of-thought/2014/06/23/13c9ac88f8a1-11e3-a606-946fd632f9f1_story.html

19. Stark E, Abeles M. (2007) Predicting movemen from multiunit activity. J. Neurosci. 27:8387-94.

20. Choi YS, Koenig MA, Jia X, Thakor NV. (2010). Quantifying time-varying multiunit neural activity using entropy-based measures. IEEE Trans. Biomed. Eng. 57:2771-7.

21. Bansal AK, Truccolo W, Vargas-Irwin CE, Donoghue JP. (2012) Decoding 3D reach and grasp from hybrid signals in motor and premotor cortices: spikes, multiunit activity, and local field potentials. J. Neurophysiol. 107:1337-55.

22. Georgopoulos AP, et al. (2007). Synchronous neural interactions assessed by magnetoencephalography: a functional biomarker for brain disorders. J. Neur. Engin. 4:349.

23. Krames ES, Peckham PH, Rezai AR (eds.). (2009). Neuromodulation. Boston: Elsevier Academic Press. 2 vols.

24. Tepper SJ, et al. (2009). Acute treatment of intractable migraine with sphenopalatine ganglion electrical stimulation. Headache. 49:983-9.

25. Peckham PH, Creasey GH. (1992). Neural prostheses: clinical applications of functional electrical stimulation in spinal cord injury. Spinal Cord. 30:96-101.

26. Sun FT, Morrell MJ, Wharen Jr RE. (2008). Responsive cortical stimulation for the treatment of epilepsy. Neurotherapeutics. 5:68-74.

27. Li M, et al. (2004). Vagal nerve stimulation markedly improves long-term survival after chronic heart failure in rats. Circulation. 109:120-4.

28. Greenberg BD, et al. (2006). Three-year outcomes in deep brain stimulation for highly resistant obsessive-compulsive disorder. Neuropsychopharmacology. 31:2384-93.

29. Deuschl G et al. (2006). A randomized trial of deep-brain stimulation for Parkinson's disease. New Eng. J. Med. 355:896-908.

30. Laxton AW, et al. (2010). A phase I trial of deep brain stimulation of memory circuits in Alzheimer's disease. Ann. Neurol. 68:521-34.

31. Racine E, Waldman S, Palmour N, Risse D, Illes J. (2007). "Currents of hope": neurostimulation techniques in US and UK print media. Camb. $Q$. Healthc. Ethics. 16:312-6.

32. Tracey, KJ. (2002). The inflammatory reflex. $\mathrm{Na}$ ture. 420:853-9.

33. Borovikova LV, et al. (2000) Vagus nerve stimula- tion attenuates the systemic inflammatory response to endotoxin. Nature. 405:458-62.

34. Rosas-Ballina M, et al. (2011) Acetylcholine-synthesizing $\mathrm{T}$ cells relay neural signals in a vagus nerve circuit. Science. 334:98-101.

35. Rosin B, et al. (2011). Closed-loop deep brain stimulation is superior in ameliorating parkinsonism. Neuron. 72:370-84.

36. Elger CE, Lehnertz K. (1998). Seizure prediction by non?linear time series analysis of brain electrical activity. Eur. J. Neurosci. 10:786-9.

37. Greene BR, et al. (2008). A comparison of quantitative EEG features for neonatal seizure detection. Clin. Neurophysiol. 119:1248-61.

Cite this article as: Bouton C. (2015) Neural decoding and applications in bioelectronic medicine. Bioelectron. Med. 2:20-4. 